\title{
Atomic-scale characterisation of catalyst nanoparticles in ionic liquids by atom probe tomography
}

\author{
Tong $\mathrm{Li}^{1}$, Hayo Meyer ${ }^{1}$ and Alfred Ludwig ${ }^{1}$
}

1. Institute for Materials \& Zentrum für Grenzflächendominierte Höchstleistungswerkstoffe (ZGH), Ruhr-Universität Bochum, Universitätsstraße 150, 44780 Bochum, Germany

* Corresponding author: tong.li@rub.de

Heterogeneous catalyst nanoparticles have proven remarkably successful in catalysing a wide range of important processes, in fuel-cells, exhaust emission control and water electrolysers. The size, structure and composition of the catalyst nanoparticles have a strong influence on the catalytic selectivity, activity and stability. To optimise the performance of catalyst materials in these devices, researchers urgently require the means for detailed characterisation to improve the understanding of relationships between the synthesis, structure and performance. Although a range of powerful analytical methods, including surface techniques, chemical spectroscopy and electron microscopy can provide a great deal of valuable information, identifying three-dimensional location of individual atoms of the catalyst nanoparticles remains a challenge. This knowledge will be required to produce the improved catalysts needed for future energy- and materials-efficient technologies.

Atom probe tomography (APT) offers a unique method for resolving chemical identities of individual atoms, with near-atomic resolution, in three-dimensions [1]. Our previous work on the characterisation of core-shell nanoparticles by APT [2] has demonstrated that this technique has an exciting potential to improve the understanding of structure-synthesis-performance relationships. Despite success with these studies [2, 3], the characterisation of catalyst nanoparticles by APT is still challenging due to difficulties associated with specimen preparation that plays a crucial role in the success rate of the subsequent APT experiments and data quality. In the previous studies $[2,3]$, an electrophoresis method was applied to draw the nanoparticles onto a blunt metal tip by electric-field. Here, we use a simple approach to deposit the nanoparticles embedded in an ionic liquid on a pre-cut Si microtip post. The catalyst nanoparticles (Mo) were prepared by inert sputter deposition into an ionic liquid ([Hdmim][(Tf)2N]) which can prevent the agglomeration of the nanoparticles. The Mo nanoparticle suspension is firstly heated up to $80^{\circ} \mathrm{C}$ at which the ionic liquid transforms to a liquid state as the melting point of [Hdmim] [(Tf) $\left.2 \mathrm{~N}\right]$ is $49^{\circ} \mathrm{C}$ [4]. A drop of the pre-heated ionic liquid is placed on the Si microtip post in which a cavity with a size $150 \mathrm{~nm}$ in diameter and $500 \mathrm{~nm}$ in depth was created by focused ion beam (FIB), as shown in Fig 1a-b. After the deposition, the nanoparticle suspension in the Si microtip post turns to a solid state at room temperature. The preliminary data in Figure 1c shows that the Mo nanoparticles with a size of 1-2 $\mathrm{nm}$ are present in the ionic liquid. These preliminary results provide evidence of the feasibility of the new approach to characterise catalyst nanoparticles by APT [5]. 
References:

[1] T. Li, O. Kasian, S. Cherevko, S. Zhang, S. Geiger, C. Scheu, P. Felfer, D. Raabe, B. Gault, K. J. J. Mayrhofer, Nature Catalysis 1 (2018), p. 300.

[2] K. Tedsree, T. Li, S. Jones, C. W. A. Chan, K. M. K. Yu, P. A. Bagot, E. A. Marquis, G. D. Smith, S. C. E. Tsang, Nature nanotechnology 6 (2011), p. 302.

[3] T. Li, P. A. Bagot, E. Christian, B. R. Theobald, J. D. Sharman, D. Ozkaya, M. P. Moody, S. E. Tsang, G. D. Smith, ACS Catalysis 4 (2014), p. 695.

[4] G. García, M. Atilhan, S. Aparicio, Physical Chemistry Chemical Physics 17 (2015), p. 13559.

[5] The authors acknowledge funding from the Deutsche Forschungsgemeinschaft (DFG, German Research Foundation) - Projektnummer 388390466 - TRR 247 (C4).

a)

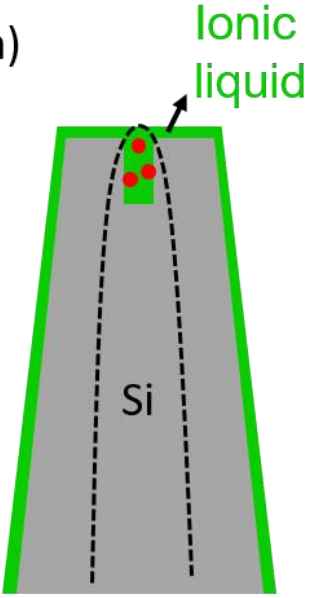

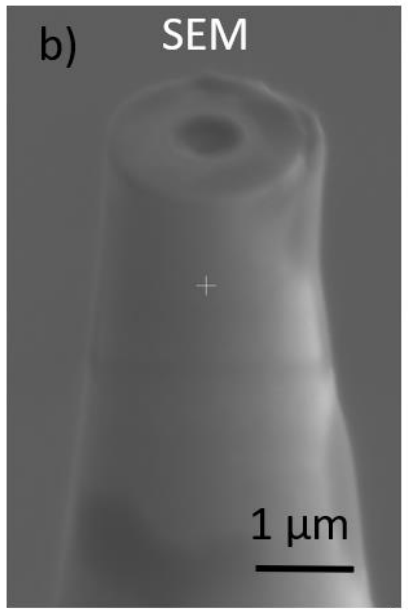

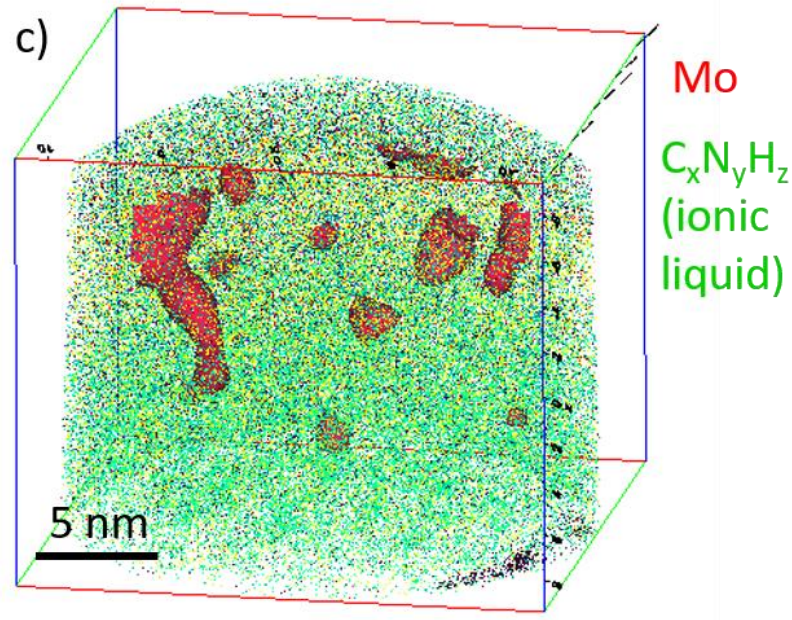

Figure 1. a) schematic diagram, b) SEM image showing the nanoparticle suspension (in solid state) covers the Si microtip post, and c) APT reconstruction revealing that Mo nanoparticles (red) are present in the ionic liquid (green). 